\title{
Le syndrome de Prader-Willi en 2015
}

Maïthé Tauber ${ }^{1}$, Denise Thuilleaux ${ }^{2}$, Éric Bieth ${ }^{3}$

> Le syndrome de Prader-Willi est un trouble du développement lié à un défaut d'expression de gènes de la région $15 q 11-q 12$ soumise à une empreinte parentale maternelle. Le diagnostic peut aujourd'hui être porté très tôt, ce qui permet de décrire l'histoire de la maladie et, en particulier, les phases nutritionnelles allant de difficultés alimentaires sévères en période néonatale jusqu'à l'apparition d'une obésité morbide avec hyperphagie et déficit de satiété. S'associent des dysfonctionnements endocriniens, un déficit cognitif modéré, des troubles des apprentissages, des troubles du comportement et des habilités sociales, et des troubles psychiatriques. La prise en charge multidisciplinaire a été fortement développée grâce à la mise en place du plan Maladies rares en 2004 et du plan Obésité en 2011, en lien avec l'association des familles (PraderWilli France). Des perspectives thérapeutiques physiopathologiques intéressantes existent aujourd'hui. <

Le syndrome de Prader-Willi (SPW), décrit en 1956 par Prader, Labhart et Willi [1], est un trouble complexe du développement d'origine génétique (touchant 1 pour 15 à 20000 naissances), lié à un défaut d'expression de certains gènes de la région chromosomique $15 q 11$ q12 [2]. C'est un modèle de maladie liée à l'empreinte

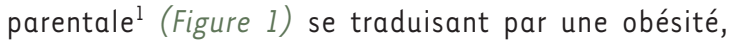
des anomalies endocriniennes et métaboliques et des troubles psychiatriques $[27](\rightarrow)$.

\section{$(\rightarrow)$ Voir la Nouvelle de P. Monnier et L. Dandolo, $m / s n^{\circ} 1$, janvier 2013, page 19}

${ }^{1}$ Empreinte parentale : chez tous les mammifères, les génomes mâle et femelle qui se rencontrent dans l'oeuf fécondé sont marqués d'un sceau différent, nommé empreinte. Par la suite, l'identification de gènes spécifiques soumis à empreinte parentale a permis de montrer que cette empreinte conduit à une expression monoallélique, dépendante de l'origine parentale. Les caractéristiques moléculaires de ce phénomène de marquage épigénétique ont maintenant été décrites et permettent d'expliquer certaines maladies humaines liées à des gènes soumis à empreinte.

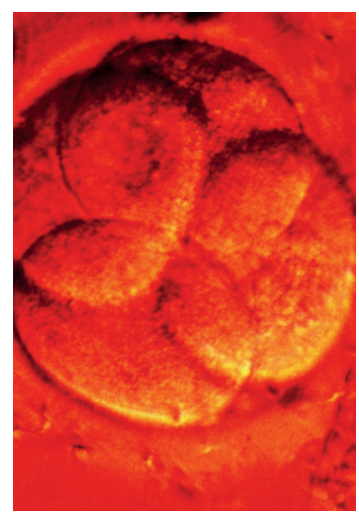

${ }^{1}$ Centre de référence du SPW, unité d'endocrinologie, obésité, maladies osseuses, génétique et gynécologie médicale, hôpital des enfants, CHU de Toulouse, 330 avenue de Grande-Bretagne, TSA 70034, 31059 Toulouse Cedex 9 , France ;

${ }^{2}$ Unité Prader-Willi, hôpital marin de Hendaye, Hendaye, France;

${ }^{3}$ Génétique médicale, hôpital Purpan, CHU de Toulouse, France. tauber.mt@chu-toulouse.fr

Depuis plus de 10 ans, le diagnostic fait précocement, dès les premiers mois de vie, a permis de décrire plus finement l'histoire de la maladie et sa complexité, en particulier, les différentes phases nutritionnelles [3]. Le phénotype néonatal associe une hypotonie sévère et des difficultés de succion/déglutition pouvant conduire à un important déficit de prise de poids et justifiant une nutrition par sonde nasogastrique dans $80 \%$ des cas. Il existe un syndrome dysmorphique modéré qui se caractérise par des yeux en amande, une bouche en chapeau de gendarme, une lèvre supérieure fine et une acromicrie ${ }^{2}$ (Figure 2). En l'absence de prise en charge adaptée et efficace, on observe toujours l'apparition d'une prise de poids excessive qui entraîne une obésité sévère avec hyperphagie et déficit de satiété, associée à des dysfonctionnements endocriniens multiples (en lien avec une pathologie hypothalamique), de nombreuses comorbidités, un déficit cognitif modéré, des troubles des apprentissages, du comportement et des habilités sociales et, dans certains cas, des troubles psychiatriques spécifiques. Ce tableau, variable au cours de la vie et d'un individu à l'autre, est fortement influencé par l'environnement et la prise en charge, et explique les conséquences sévères de cette maladie pour les patients, les familles, et les soignants ainsi que la morbidité et la mortalité élevées [2,4].

\section{Diagnostic}

Bien que le diagnostic soit facilement suspecté sur des critères cliniques, variables au cours de la vie, le test génétique est indispensable pour le confirmer. Plusieurs méthodes peuvent être utilisées. L'analyse de la méthylation de I'ADN devrait être réalisée en première intention, car elle permet de confirmer le diagnostic dans

${ }^{2}$ L’acromicrie décrit l'existence de petites extrémiés (mains et pieds). 


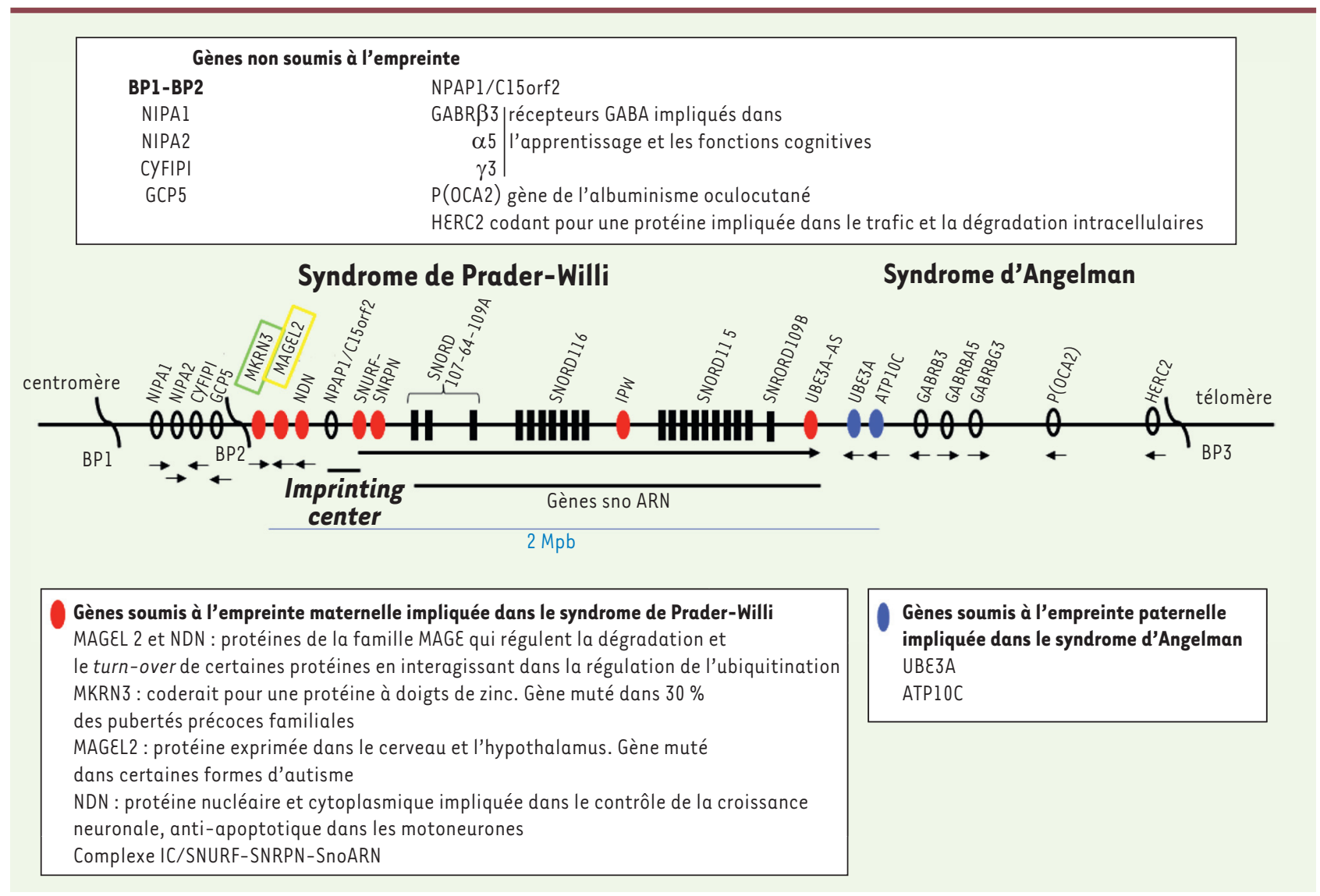

Figure 1. La région chromosomique 15q11-q12. Grande région comprenant des gènes soumis à empreinte maternelle, candidats pour le SPW (ovales rouges), des gènes soumis à empreinte paternelle impliqués dans le syndrome d’Angelman (ovales bleus) et des gènes non soumis à empreinte (ovales noirs vides), avec par conséquent une expression biallélique dont le rôle dans les différences phénotypiques en fonction du génotype n’est pas exclu.

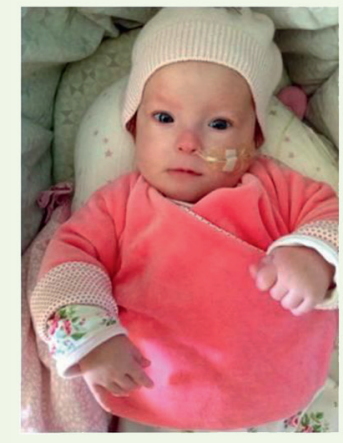

Front étroit

Yeux en amandes

Lèvre supérieure fine

Bouche en chapeau de gendarme

Fille âgée de 6 mois, disomie

Sonde nasogastrique pendant 6,5 mois

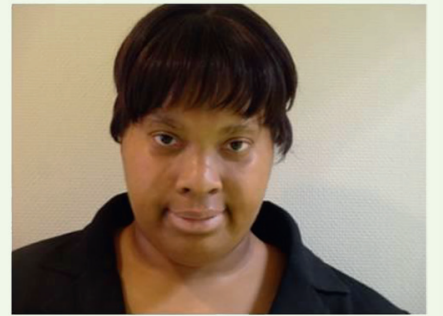

Fille de 20 ans, délétion, taille 1,65 m IMC $55,8 \mathrm{~kg} / \mathrm{m}^{2}$

Troubles du comportement avec opposition, recherche de nourriture +++, trichotillomanie (trouble caractérisé par l'arrachage compulsif de ses propres poils et/ou cheveux)

$\mathrm{Pl}=47$

Figure 2. Dysmorphie faciale. Deux photos du visage de filles atteintes du SPW montrant les signes dysmorphiques faciaux que l'on retrouve chez le jeune enfant et chez l'adulte. Sont également notées les caractéristiques cliniques.

Une fois le diagnostic de SPW confirmé, il est indispensable de préciser le mécanisme génétique responsable afin d'assurer un conseil génétique et de mettre en place une prise en charge. Les différents types génétiques et les méthodes généralement utilisées pour en faire le diagnostic, sont

la presque totalité des cas, en mettant en évidence la seule présence de l'allèle méthylé d'origine maternelle (allèle soumis à l'empreinte parentale) tandis que l'allèle non méthylé d'origine paternelle (allèle fonctionnellement actif) est absent. Ainsi, un profil normal de méthylation de la région $15 q 11-q 12$ exclut à $99 \%$ le diagnostic de syndrome de Prader-Willi. présentés dans le Tableau I.

\section{Le diagnostic différentiel se pose dans différentes} situations

- On élimine facilement d'autres obésités syndromiques associant une déficience intellectuelle. 


\begin{tabular}{|c|c|c|c|c|}
\hline Type génétique & Fréquence & Méthode & Précautions & $\begin{array}{l}\text { Transmission/risque } \\
\text { de récurrence }\end{array}$ \\
\hline $\begin{array}{l}\text { Délétion chromosome } \\
\text { paternel }\end{array}$ & $60 \%-65 \%$ & $\begin{array}{l}\text { Méthylation } \\
\text { FISH (SNRPN)* } \\
\text { PMPSF/MLPA }\end{array}$ & $\begin{array}{l}\text { Si FISH négatif, } \\
\text { poursuivre l'analyse géné- } \\
\text { tique (méthylation) }\end{array}$ & $\begin{array}{l}\text { NON/pas supérieur à celui } \\
\text { de la population générale }\end{array}$ \\
\hline Type 1 & $30 \%$ & Typage de la délétion par & Laboratoires spécialisés & \\
\hline Type 2 & $60 \%$ & QMPSF/MLPA & nécessaires & \\
\hline Atypiques & $10 \%$ & CGH array & & \\
\hline Disomie unimaternelle & $\begin{array}{l}33-35 \% \\
\text { Actuellement } \uparrow \\
\text { (du fait de l'âge } \\
\text { maternel) }\end{array}$ & $\begin{array}{l}\text { Méthylation } \\
\text { Analyse de liaison (étude } \\
\text { de la ségrégation } \\
\text { de marqueurs) }\end{array}$ & $\begin{array}{l}\text { Étude des } 2 \text { parents } \\
\text { nécessaire }\end{array}$ & $\begin{array}{l}\text { NON/pas supérieur à celui } \\
\text { de la population générale }\end{array}$ \\
\hline $\begin{array}{l}\text { Dérèglement du centre } \\
\text { de l'empreinte (IC) }\end{array}$ & $2-5 \%$ & & & OUI/NON \\
\hline Délétion de I’IC & $\begin{array}{l}30 \%(1-2 \% \\
\text { du total) }\end{array}$ & $\begin{array}{l}\text { Méthylation } \\
\text { MLPA/QMPSF } \\
\text { CGH array }\end{array}$ & & $\begin{array}{l}\text { OUI/si délétion, IC présente } \\
\text { chez le père, récurrence } \\
50 \%\end{array}$ \\
\hline $\begin{array}{l}\text { Épimutation primaire } \\
\text { (épigénétique) }\end{array}$ & $\begin{array}{l}70 \%(2-3 \% \\
\text { du total })\end{array}$ & $\begin{array}{l}\text { Aucune (diagnostic } \\
\text { d'élimination) }\end{array}$ & & $\begin{array}{l}\text { NON/pas supérieur à celui } \\
\text { de la population générale }\end{array}$ \\
\hline $\begin{array}{l}\text { Translocation, ou rares } \\
\text { remaniements } \\
\text { (marqueurs, inversions) } \\
\text { impliquant la région } \\
15 \text { qliql2 }\end{array}$ & $<1 \%$ & $\begin{array}{l}\text { Caryotype }{ }^{\star \star} \\
\text { Méthylation (négative } \\
\text { pour certaines } \\
\text { translocations) }\end{array}$ & & $\begin{array}{l}\text { OUI/variable selon } \\
\text { I'anomalie (peut atteindre } \\
10 \% \text { si translocation, } \\
\text { et impliquer d'autres } \\
\text { chromosomes) }\end{array}$ \\
\hline
\end{tabular}

Tableau I. Différents types génétiques de SPW. * Attention, si le résultat de la FISH, utilisée en première intention est négatif, il faut poursuivre les investigations par une étude de la méthylation de SNRPN (small nuclear ribonucleoprotein polypeptide N) afin d'éviter un « faux négatif ». ** Le caryotype de l'enfant et des parents reste nécessaire pour analyser la morphologie des chromosomes 15 et mettre en évidence de rares remaniements complexes (translocations ou inversions héritées). DMPSF : quantitative multiplex PCR of short fragments; MLPA : multiplex ligation-dependent probe amplification ; IC : imprinting center (centre de l'empreinte).

- Si le tableau clinique est fortement évocateur de SPW, il faudra adresser le patient à une équipe spécialisée pour la recherche de mutations de certains gènes dans la région SPW (en particulier des mutations des gènes SNORD116 et MAGEL2) ou de délétions/disomies dans d'autres régions chromosomiques (chromosome 14q32, translocation impliquant le chromosome 3 ou le chromosome 6). On a aujourd'hui tendance à parler de SPW-like pour toutes les obésités syndromiques. Toutefois, à notre avis, cette dénomination ne se justifie pas, car elle entraîne une confusion et appauvrit la description des phénotypes.

\section{L'annonce du diagnostic}

Elle est aujourd'hui faite le plus souvent en période néonatale, ou chez des adultes chez lesquels le diagnostic n'avait pas été posé. Elle doit se faire en période néonatale, en présence d'un médecin connaissant le syndrome ; le pédiatre endocrinologue doit également rapidement rencontrer la famille.

Le diagnostic prénatal peut être établi sur des signes d'appel associant hydramnios du dernier trimestre, immobilisme fœtal, et certaines anomalies positionnelles des mains et des pieds. À notre avis, le SPW est une pathologie qui, aujourd'hui, répond aux critères permettant de proposer une interruption médicale de grossesse.

\section{Un phénotype clinique complexe et évolutif}

\section{Les phases nutritionnelles et le comportement} alimentaire

Le diagnostic précoce, fait au cours du premier mois de vie, a permis de décrire différentes phases nutritionnelles $[3,5]$. De la naissance à 2 ans, le tableau clinique est marqué par l'hypotonie sévère, les troubles de la succion/déglutition et les faibles interactions d'un bébé très peu sollicitant, amimique, suivant peu du regard et ne pleurant pas, ou plutôt pleurant sans bruit. Ces bébés ont peu de force et manquent de coordination pour téter, mais nous pensons de plus en plus qu'il s'agit plutôt d'une anorexie 
particulière du nourrisson. Exceptionnels sont ceux qui peuvent téter le sein de leur mère.

En l'absence de prise en charge spécifique, il y a une prise de poids précoce excessive et rapide. De manière intéressante, cette prise de poids précède l'hyperphagie et le défaut de satiété, deux caractéristiques du tableau classique chez l'enfant et l'adolescent, qui persistent à l'âge adulte, et qui entraînent une obésité morbide avec ses complications respiratoires, métaboliques, cardiovasculaires et orthopédiques.

\section{Une obésité particulière}

L'excès de masse grasse chez les personnes présentant un SPW est essentiellement sous-cutané. Le tissu adipeux est particulier chez l'enfant, avec un déficit de précurseurs adipocytaires (ou cellules souches), et chez l'adulte, peu de fibrose. II semble que l'expansion du tissu adipeux puisse se faire jusqu'à des limites extrêmes, avec un profil métabolique en général meilleur que celui des personnes obèses de corpulence identique [6]. Le traitement par l'hormone de croissance permet de restaurer, au moins partiellement, certaines propriétés du tissu adipeux, avec chez l'enfant, une normalisation du nombre de précurseurs adipocytaires. Ces effets pourraient persister après l'arrêt du traitement [7]. De plus, ces patients ont une sensibilité accrue à l'insuline, comparativement à des personnes de même indice de masse corporelle (IMC) et de même adiposité. Cependant, si l'IMC est très important, un diabète de type 2 apparaît dans $25 \%$ des cas environ, probablement parce qu'il existe également une insulinopénie [8].

\section{Des dysfonctionnements endocriniens multiples}

L'anomalie hypothalamique, bien que non élucidée sur le plan physiopathologique, est bien démontrée tant chez l'homme, par les études histologiques et endocriniennes, que dans des modèles animaux (souris). De plus, les études d'imagerie fonctionnelle cérébrale montrent qu'il existe des anomalies très variées de perfusion et de métabolisme qui ne touchent pas seulement l'hypothalamus. II existe en effet de nombreuses altérations dans le réseau neuronal régulant l'appétit, qui implique en particulier l'hypothalamus, le cingulum antérieur, le cervelet, le cortex préfrontal et l'insula [9-11]. II semblerait que ce soit surtout un défaut de contrôle de la prise alimentaire qui sous-tende le comportement alimentaire.

\section{Un déficit somatotrope particulier}

À l'inverse de ce qui existe dans l'obésité commune, un réel déficit en hormone de croissance est observé chez $88 \%$ des enfants présentant un SPW [12, 13], avec, chez tous ces enfants, des taux bas d'IGF-1 (insulin-like growth factor 1). Le traitement par l'hormone de croissance est autorisé en Europe et aux États-Unis depuis 2000, en raison de ses effets positifs sur la croissance et la composition corporelle. Plus le traitement est commencé précocement (au cours de la première année de vie), meilleurs sont les effets sur le développement psychomoteur et cognitif [14]. Les enfants présentant un SPW semblent avoir une sensibilité accrue au traitement, comme en témoignent les taux élevés d'IGF-1 sous hormone de croissance [15]. Avec un recul de 8 ans de traitement par l'hormone de croissance, on peut aujourd'hui affirmer que le rapport bénéfice/risque est positif pour ces patients, et que ce traitement a permis de transformer leur vie d'enfant, d'adolescent et d'adulte [16].

L'hormone de croissance n'a pas d'autorisation de mise sur le marché (AMM) pour le traitement d'adultes atteints de SPW, malgré l'effet positif sur la composition corporelle et les fonctions cognitives rapporté dans la littérature [17].

\section{Un hypogonadisme « mixte »}

La description clinique et paraclinique de l'hypogonadisme a été récemment précisée [18-21]. Chez le garçon, il s'exprime dès le début de la vie par la présence quasi constante d'une ectopie testiculaire et, moins fréquemment, d'un pénis peu développé. Chez la fille, il se traduit par une hypoplasie des petites lèvres, peu évidente à diagnostiquer. II semble que la minipuberté ${ }^{3}$ soit présente [22] et qu'il existe un continuum d'anomalies allant de l'hypogonadisme hypogonadotrope pur, à l'hypogonadisme périphérique presque pur, en passant par des dysfonctions mixtes, tant chez les hommes que chez les femmes. La puberté est rarement complète justifiant une supplémentation hormonale pour l'induction ou le maintien des caractères sexuels secondaires.

Il existe de rares situations de puberté précoce vraie chez certains enfants avec un SPW. Cela est à mettre en relation avec la mise en évidence de mutations du gène MKRN3 (makorin ring finger protein 3$)^{4}$ situé dans la région chromosomique SPW responsables de certaines pubertés précoces familiales [23]. À côté de ce gène qui inhibe le début pubertaire, le gène NDN, également dans la région chromosomique SPW, et codant la necdine, est également impliqué dans certains cas d'hypogonadisme central, faisant du SPW un modèle précieux pour l'étude du développement pubertaire.

Les autres atteintes endocriniennes sont décrites dans le Tableau II.

\section{Des aspects psychologiques et psychiatriques particuliers}

\section{Les troubles du comportement alimentaire}

Les troubles du comportement alimentaire sont constants. Leur intensité varie entre les personnes et

\footnotetext{
${ }^{3}$ Activation très précoce et transitoire de l'hypothalamus dans les premiers mois de vie.

${ }^{4}$ MKRN3 est un gène exprimé par le chromosome paternel. II est soumis à empreinte et est localisé dans la région 15ql1-q12.
} 
Prise en charge de l'obésité et des anomalies antéhypophysaires

- Obésité, aujourd'hui < $50 \%$ chez l'enfant et $70 \%$ chez l'adulte

- leptine $\uparrow$, ghréline $\uparrow$, polypeptide pancréatique $\downarrow$

- $\downarrow$ du nombre et du volume des neurones à ocytocine dans le noyau para-ventriculaire hypothalamique

- Diabète de type 2, rare chez l'enfant, $25 \%$ chez l'adulte si obèse

- Déficit en hormone de croissance, > $80 \%$ des patients, $25 \%$ nés petits pour l'âge gestationnel

- Hypogonadisme, mixte garçons et filles $>60 \%$

- Cryptorchidie, $80-90 \%$, petit pénis $30 \%$; chirurgie de la cryptorchidie

- Hypothyroïdie, 20 à $80 \%$

- Insuffisance surrénalienne centrale, 10 à $60 \%$

\section{Dépistage et prise en charge des comorbidités}

- Troubles respiratoires du sommeil, apnées (consultation ORL, amygdalectomie et adénoïdectomie si nécessaire, pas systématiques), troubles du sommeil \pm endormissement diurne excessif \pm narcolepsie, catalepsie, catatonie

- Évaluation des troubles dysautonomiques en fonction des signes cliniques

- Énurésie fréquente

- Scoliose, 30 à $80 \%$, début à un âge moyen de 7 ans, $20 \%$ avant 5 ans; chirurgie dans 15 à $20 \%$ des cas

- Épilepsie, $10 \%$ environ

- Troubles digestifs : constipation, ballonnement, diarrhées, reflux gastro-oesophagien

- Troubles ophtalmologiques : myopie, hypermétropie plus fréquentes, strabisme, voir si orthoptie nécessaire

- Bilan dentaire et orthodontique ; troubles de l'émail améliorés si alimentation contrôlée et bonne hygiène

- Lésions de grattage cutanéomuqueux : traitement et prévention

\section{Évaluations régulières et rééducations}

- Prise en charge orthophonique, oralité, pragmatique

- Évaluation des fonctions cognitives, évaluation des troubles d'apprentissages

- Évaluation de l'environnement social

- Orientation scolaire et projet de vie

Évaluations répétées des troubles du comportement et des troubles psychiatriques : suivi et prise en charge adaptés \pm psychotropes

Tableau II. Obésité, anomalies endocriniennes et prise en charge.

selon l'état psychologique du patient présentant un SPW. Ces patients ont un comportement alimentaire compulsif et une préoccupation prégnante pour la nourriture (plus qu'une faim permanente) qui peut s'atténuer dans un environnement structuré et si l'on stimule d'autres intérêts. Ils ont une appétence marquée pour le sucre, et un rassasiement retardé avec une faible satiété. Ils anticipent le repas avec une précision impressionnante sur l'horaire, et se montrent très compétents lorsqu'ils savent que de la nourriture peut être disponible et dérobée. Dans ces situations, malgré des fonctions exécutives déficientes, ils sont capables de développer des stratégies et une planification remarquables pour aller dérober et consommer cette nourriture, y compris en quantités très importantes. Dans ces cas, les risques de fausse route et de rupture gastrique ne sont pas négligeables. Les ruptures gastriques rapportées, qui sont favorisées par une consommation alimentaire très importante et très rapide, sont liées à une fragilité de la muqueuse gastro-intestinale et à des troubles de la motricité digestive qui restent mal connus.

\section{Le déficit cognitif et les difficultés d'apprentissage}

Tous les patients atteints du SPW présentent des troubles cognitifs et la plupart ont un déficit intellectuel d'intensité légère ou moyenne.
II existe cependant une grande variabilité entre les patients, pouvant aller d'un retard léger, avec de bonnes capacités, y compris verbales, et un bon niveau d'études jusqu'à un retard grave, avec pauvreté ou absence de langage. On retrouve également une rigidité mentale, une absence de fluidité de la pensée et des difficultés de conceptualisation. Des déficits neurologiques sont présents. Ils touchent l'attention, la mémoire, les fonctions exécutives, et sont associés à des difficultés avec le temps, l'espace et la causalité. Quel que soit leur niveau, la capacité d'adaptation des patients reste faible et leur intégration en milieu ordinaire s'avère donc difficile, voire impossible.

\section{Un trouble des habilités sociales et de la pragmatique}

Il existe un déficit de la théorie de l'esprit (capacité d'inférer l'esprit de l'autre) en lien étroit avec l'empathie. La pragmatique est complexe pour ces personnes, avec une absence de sens figuré, une difficulté d'abstraction justifiant l'importance de faire passer des messages par l'écrit ou le visuel. Ces troubles décrits 


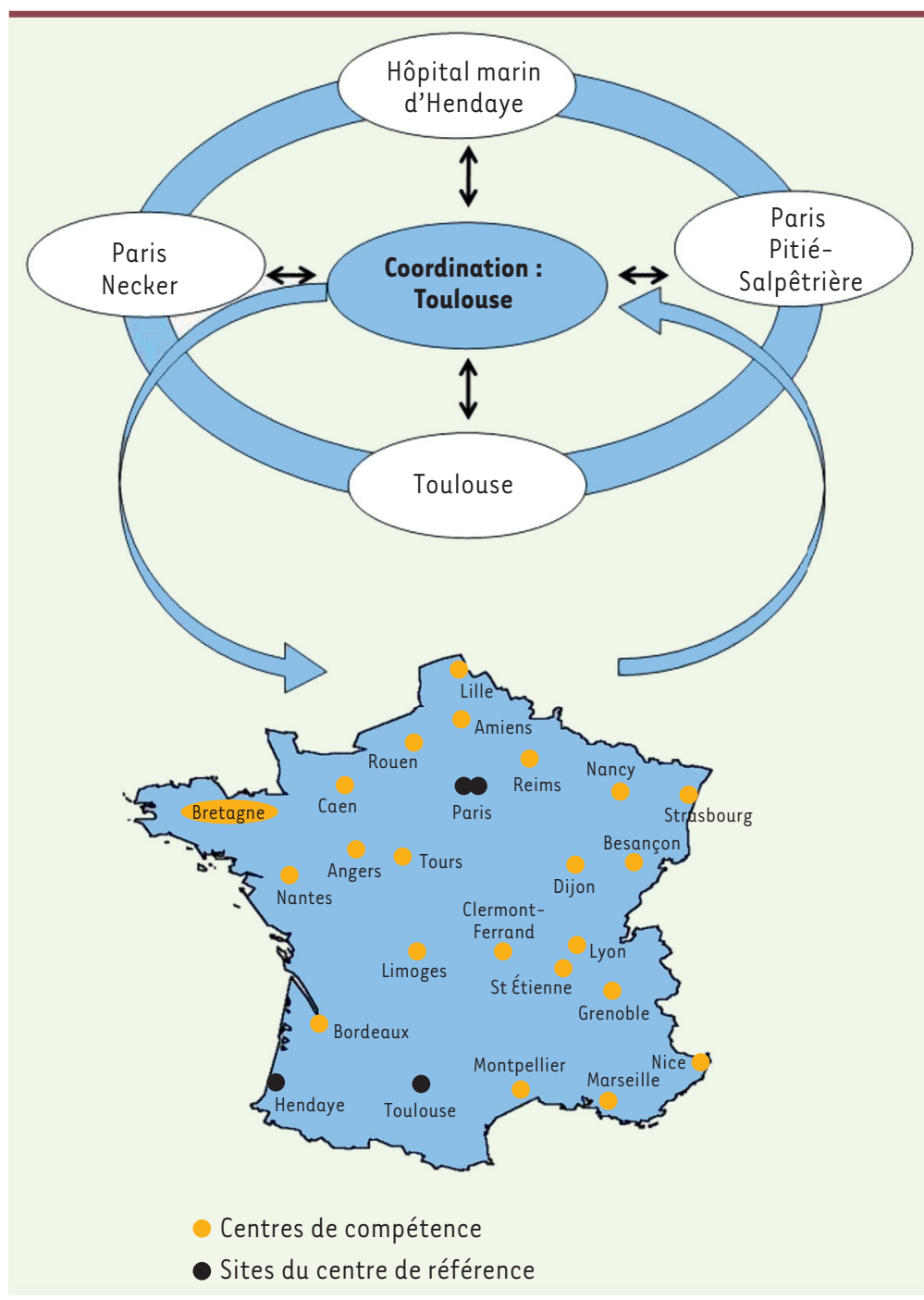

Figure 3. Organisation de la prise en charge territoriale. Le plan Maladies rares a permis de labelliser le centre de référence du SPW en 2004 avec ses 4 sites (ronds noirs, le CHU de Toulouse, l'hôpital Necker, l'hôpital de la Pitié-Salpêtrière et l'hôpital marin d'Hendaye, la coordination se trouve au CHU de Toulouse). Le centre de référence coordonne aussi les centres de compétence (ronds oranges).

dans le spectre autistique, aujourd'hui considérablement élargi, sont présents dans le SPW. De manière intéressante, on a récemment décrit une mutation du gène MAGEL2 chez des patients atteints d'autisme et ayant également des troubles alimentaires précoces.

\section{Les troubles du comportement}

Des troubles du comportement peuvent se manifester à tout âge, avec une aggravation à l'adolescence et chez le jeune adulte. Ces troubles ne sont pas uniquement la conséquence du déficit cognitif.

Les classifications nosographiques habituelles (regroupées dans les DSM-IV et $V$ et la $(I M-10)^{5}$ ne décrivent pas de manière adaptée les

${ }^{5}$ DSM : Manuel diagnostique et statistique des troubles mentaux. CIM : Classification internationale des maladies. troubles psychiatriques de ces patients. Le profil comportemental et psychopathologique est modulé selon le génotype (délétion ou non-délétion), ce qui justifie son étude systématique. II est également influencé par les relations intrafamiliales, l'interaction sociale et l'éducation, ce qui justifie une évaluation psychosociale et psychiatrique systématique et répétée.

Ces patients présentent, dans la grande majorité des cas, des conduites d'automutilation (grattage) touchant la peau ou l'ensemble des muqueuses (anale, vaginale, nasale).

Les troubles du comportement et les troubles psychiatriques sont souvent la première cause de la diminution de la qualité de vie de ces patients et de leur famille. L'intégration et l'adaptation sociale sont rendues difficiles empêchant une autonomie de vie complète. À l'âge adulte, une protection juridique est presque toujours nécessaire.

Ces patients présentent aussi des troubles psychoaffectifs avec une labilité émotionnelle, une immaturité et une grande instabilité. Ils sont très attachants, « sociables mais non socialisables ».

\section{Les différentes atteintes organiques}

Le Tableau /I résume les différentes atteintes qui rendent compte de la complexité du syndrome de Prader-Willi. La prise en charge est guidée par les évaluations répétées chez les patients tout au long de leur vie.

\section{La prise en charge}

\section{Une organisation des soins et un maillage territorial complémentaire}

Le plan Maladies rares a labellisé le centre de référence du SPW multisites (Figure 3). La publication par la Haute autorité de santé du programme national de diagnostic et de soins ${ }^{6}$ en 2012, a permis de décrire la prise en charge multidisciplinaire dans le centre de référence et les centres de compétence qui sont répartis sur le territoire, en relation avec le médecin traitant. En 2011, le plan Obésité a labellisé deux établissements de soins de suite et de réadaptation pour enfants (un en Île-de-France et un près de Toulouse), en lien avec les sites des centres de référence, pour l'accueil des enfants atteints de SPW et d'autres obésités syndromiques ou apparentées. Des

\footnotetext{
${ }^{6}$ http://www.has-sante.fr/portail/jcms/c_1216145/ald-hors-liste-syndrome-deprader-willi
} 
recommandations [24] ont été écrites dans le cadre de la prise en charge nutritionnelle et de l'activité physique des personnes en situation de handicap mental.

La clé de la prise en charge est la prise en compte réelle de l'intrication somatique et psychique de cette pathologie et le respect de la personne. Il s'agit de soigner avec expertise et bienveillance, et d'accompagner au quotidien la personne et son entourage, en intégrant les dimensions endocriniennes et psychiques, les multiples comorbidités, le déficit cognitif, les difficultés émotionnelles, les déficits des habilités sociales et de la pragmatique, et cela de manière holistique. L'objectif est de trouver un équilibre raisonnable entre un suivi basé sur la connaissance de la maladie et la protection de la qualité de vie de ces personnes.

Il s'agit, comme le dit le président de l'association Prader-Willi France, François Besnier, «de prendre du recul par rapport au syndrome et de se rapprocher de la personne ». En France, 50 \% des adultes présentant un SPW vivent au domicile des parents car il n'y a pas encore assez de lieux parfaitement adaptés, en particulier, en ce qui concerne l'accès à l'alimentation. En partenariat avec le centre de référence, l'association Prader-Willi France, grâce à un financement de la caisse nationale de solidarité pour l'autonomie, a rédigé et mis en ligne un «Guide de pratiques partagées » (http://guideprader-willi.fr/) coécrit par les familles, les soignants des centres de référence et des centres de compétence, et les établissements du secteur médicosocial.

Comme pour toute maladie chronique, de manière plus marquée lorsqu'elle débute très tôt au cours de la vie, les phases de transition sont critiques. En particulier dans le SPW, l'adolescence, du fait des changements hormonaux et des troubles psychiques qui s'aggravent à cette période, peut entraîner des ruptures dans les parcours si on n'anticipe pas suffisamment.

\section{Des associations de patients remarquables en France et dans le monde}

Les associations de patients (présentes dans 102 pays) se sont regroupées depuis de nombreuses années en une organisation internationale, I'IPWSO (international Prader-Willi syndrome organization) qui organise, depuis 1991, un congrès international auquel participent familles, soignants et chercheurs. Depuis 2003, il existe également une fondation pour la recherche sur le SPW, la FPWR (foundation for Prader-Willi research), qui collecte des fonds destinés à des bourses et à des séminaires de recherche.

\section{La recherche}

Le SPW est aujourd'hui reconnu comme un modèle de plusieurs morbidités : modèle d'obésité sévère [25], de troubles du comportement alimentaire, de troubles hypothalamiques, de troubles psychiatriques et de déficits des habilités sociales et, de manière plus générale, modèle du spectre autistique. Une réunion internationale organisée en 2009 aux NIH (national institutes of health) a eu pour objectif de lister les axes de recherche à prioriser en valorisant ceux appartenant au plan maladies rares développé en France. II s'agissait de mettre en place et de renforcer les bases de données de patients pour permettre d'écrire l'histoire naturelle de la maladie, de favoriser les collections biologiques, de développer la recherche physiopathologique, en particulier avec des modèles animaux. Les cellules souches pluripotentes (en anglais iPS pour induced pluripotent stem cells) obtenues par la « reprogrammation» de cellules de patients (souvent des fibroblastes cutanés) sont également porteuses d'espoir. Elles permettent, après différenciation, d'avoir accès au fonctionnement des neurones des patients [26]. Le développement de la recherche thérapeutique est également en expansion, avec de nouveaux produits, ou avec des molécules déjà utilisées en clinique et dont l'effet dans le SPW pourrait s'avérer bénéfique. Ces dernières années, de nombreuses désignations orphelines dans le SPW ont été approuvées par l'EMA (European medicines agency), dont l'ocytocine.

Le diagnostic précoce, les connaissances physiopathologiques et les données sur la plasticité cérébrale permettent d'envisager dans un avenir pas si lointain un traitement préventif ou modifiant substantiellement le cours de la maladie. $\diamond$

\section{SUMMARY}

\section{Prader-Willi syndrome in $\mathbf{2 0 1 5}$}

Prader-Willi syndrome is a neurodevelopmental disorder caused by the lack of expression of imprinted genes of the chromosomal region 15ql1-q12. Diagnosis can now be made in the first months of life, allowing a precise description of the natural history of the disease. Of interest, nutritional phases appear to be more complex than those initially reported, starting with a severe hypotonia with deficit of suckling and failure to thrive in neonates, and subsequently switching to excessive weight gain with morbid obesity due to hyperphagia and deficit of satiety. The phenotype also includes endocrine dysfunction, intellectual disability, learning deficits, behavioural troubles with impaired social skills and psychiatric features. Multidisciplinary care has been strongly improved by the rare disease programme launched in France in 2004 and the Obesity programme in 2011 in link with the patient association. New therapeutic perspectives have arisen from knowledge of the pathophysiology of PWS. $\diamond$

\section{LIENS D'INTÉRÊT}

Les auteurs déclarent n'avoir aucun lien d'intérêt concernant les données publiées dans cet article. 


\section{RÉFÉRENCES}

1. Prader A, Labhart A, Willi $H$. Ein syndrom von adipositas, kleinwuchs, kryptorchismus und oligophrenie nach myatonieartigem zustand im neugeborenenalter. Schweiz Med Wochenschr $1956 ; 6: 1260-1$.

2. Cassidy SB, Schwartz S, Miller JL, Driscoll DJ. Prader-Willi syndrome. Genet Med $2012 ; 14: 10-26$.

3. Miller JL, Lynch CH, Driscoll DC, et al. Nutritional phases in Prader-Willi syndrome. Am J Med Genet A 2011 ; 155A : 1040-9.

4. Goldstone AP, Holland AJ, Hauffa BP, et al. Recommendations for the diagnosis and management of Prader-Willi syndrome. J Clin Endocrinol Metab 2008 ; 93 : 4183-97.

5. Butler JV, Whittington JE, Holland AJ, et al. The transition between the phenotypes of Prader-Willi syndrome during infancy and early childhood. Dev Med Child Neurol 2010; 52 : e88-93.

6. Lacroix D, Moutel S, Coupaye M, et al. Metabolic and adipose-tissue signatures in adults with Prader-Willi syndrome, a model of extreme adiposity. J Clin Endocrinol Metab 2014; 100 : $850-9$

7. Coupaye M, Lorenzini F, Lloret-Linares C, et al. Growth hormone therapy for children and adolescents with Prader-Willi syndrome is associated with improved body composition and metabolic status in adulthood. J Clin Endocrinol Metab 2013 ; 98 : ع328-35.

8. Laurier V, Lapeyrade A, Copet $P$, et al. Medical, psychological and social features in a large cohort of adults with Prader-Willi syndrome: experience from a dedicated centre in France. J Intellect Disabil Res 2015 ; 59 : 411-21.

9. Mantoulan C, Payoux P, Diene G, et al. PET scan perfusion imaging in the Prader-Willi syndrome: new insights into the psychiatric and social disturbances. J Cereb Blood Flow Metab 2011 ; 31 : 275-82.

10. Miller JL, James GA, Goldstone AP, et al. Enhanced activation of reward mediating prefrontal regions in response to food stimuli in Prader-Willi syndrome.J Neurol Neurosurg Psychiatry 2007 ; $78: 615-9$.

11. Holsen LM, Savage CR, Martin LE, et al. Importance of reward and prefrontal circuitry in hunger and satiety: Prader-Willi syndrome vs simple obesity. Int J Obes (Lond) 2012 ; 36:638-47.

12. Diene G, Mimoun $\varepsilon$, Feigerlova $\varepsilon$, et al. Endocrine disorders in children with Prader-Willi syndrome - data from 142 children of the French database. Horm Res Paediatr 2010 ; 74 : 121-8.

13. Deal CL, Tony M, Höybye C, et al. GrowthHormone Research Society workshop summary: consensus guidelines for recombinant human growth hormone therapy in Prader-Willi syndrome.J Clin Endocrinol Metab $2013 ; 98$ ع1072-87.

14. Festen DA, Wevers $M$, Lindgren AC, et al. Mental and motor development before and during growth hormone treatment in infants and toddlers with Prader-Willi syndrome. Clin Endocrinol 2008 ; 68 919-25.
15. Feigerlova $\varepsilon$, Diene G, Oliver I, et al. Elevated insulin-like growth factor-। values in children with Prader-Willi syndrome compared with growth hormone (GH) deficiency children over two years of $\mathrm{GH}$ treatment. J Clin Endocrinol Metab $2010 ; 95: 4600-8$

16. Siemensma $\varepsilon P$, Tummers-de Lind van Wijngaarden RF, Festen DA, et al. Beneficial effects of growth hormone treatment on cognition in children with Prader-Willi syndrome: a randomized controlled trial and longitudinal study. J Clin Endocrinol Metab 2012 ; 97 : 2307-14.

17. Höybye C. Growth hormone treatment of Prader-Willi syndrome has longterm, positive effects on body composition. Acta Paediatr $2015 ; 104$ : 422-7.

18. Eldar-Geva T, Hirsch HJ, Pollak Y, et al. Management of hypogonadism in adolescent girls and adult women with Prader-Willi syndrome. Am J Med Genet A 2013 ; 161A : 3030-4

19. Eldar-Geva T, Hirsch HJ, Benarroch F, et al. Hypogonadism in females with Prader-Willi syndrome from infancy to adulthood: variable combinations of a primary gonadal defect and hypothalamic dysfunction. Eur J Endocrinol $2010 ; 162: 377-84$

20. Eldar-Geva T, Hirsch HJ, Rabinowitz R, et al. Primary ovarian dysfunction contributes to the hypogonadism in women with Prader-Willi Syndrome. Horm Res $2009 ; 72: 153-9$

21. Hirsch HJ, Eldar-Geva T, Benarroch F, et al. Primary testicular dysfunction is a major contributor to abnormal pubertal development in males with Prader-Willi syndrome. J Clin Endocrinol Metab 2009 ; 94 : 2262-8.

22. Hirsch HJ, Eldar-Geva T, Erlichman M, et al. Characterization of minipuberty in infants with Prader-Willi syndrome. Horm Res Paediatr $2014 ; 82: 230-7$.

23. Abreu AP, Dauber A, Macedo DB, et al. Central precocious puberty caused by mutations in the imprinted gene MKRN3. N Engl J Med 2013 ; 368 : 2467-75.

24. Recommandations Handicap. Obésité 2013 ; 8 ; 196-200.

25. Tauber M, Diene G, Mimoun $\varepsilon$, et al. Prader-Willi syndrome as a model of human hyperphagia. Front Horm Res 2014 ; 42 : 93-106.

26. Studer L. Derivation of dopaminergic neurons from pluripotent stem cells. Prog Brain Res $2012 ; 200: 243-63$.

27. Monnier P, Dandolo L. Rôle du locus H19 dans le placenta. Med Sci (Paris) $2013 ; 29: 19-21$

\section{TIRÉS À PART}

M. Tauber

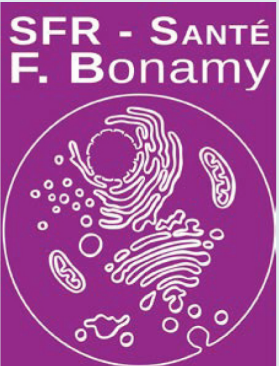

\section{Inscrivez-vous au MOOC «Ouvrez les portes du laboratoire: cellules et cellules souches 》}

Structure fédérative de recherche François Bonamy

(FED 4203/Inserm UMS 016/CNRS 3556)

Ce MOOC est le premier à associer un enseignement didactique de notions de biologie cellulaire, à un enseignement pratique, grâce à des vidéos tournées en conditions réelles au laboratoire et détaillant les principales étapes de culture et de reprogrammation de cellules souches. Grâce à ce cours, vous allez pouvoir partir à la découverte d'une plate-forme de production de cellules souches et du laboratoire de recherche de l'institut du thorax

Les inscriptions, gratuites, sont ouvertes sur le site de la plate-forme ministérielle FUN : https://www. france-universite-numerique-mooc.fr/courses/univnantes/31004S02/session02/about Les cours commenceront le lundi 7 septembre 2015 ! 